\title{
Effect of cholecystectomy on fasting lipid profile and blood glucose
}

Karki OB

Department of Surgery, Manipal College of Medical Sciences, Pokhara, Nepal

Correspondence: Dr. Om Bahadur Karki, Lecturer, Department of Surgery, Manipal College of Medical Sciences, Pokhara, Nepal

Email: Karkiom225@yahoo.com

\begin{abstract}
Background: Association between cholelithiasis and dyslipidemia has been shown in many studies. The objective of this study was to determine the changes in lipid profile and blood glucose level after cholecystectomy.

Methods: Fifty patients of cholelithiasis were studied prospectively. Total cholesterol (TC), triglycerides (TG), high density lipoprotein cholesterol (HDL-C), low density lipoprotein cholesterol (LDL-C), atherogenic index (AI) and fasting blood sugar (FBS) levels were estimated pre-operatively. Further, same parameters were studied in after cholecystectomy at one week and one month intervals. None of the patients received any lipid lowering drug or dietary restriction. Results were analysed and compared.
\end{abstract}

Results: Of the 50 patients with cholelithiasis, $68 \%$ were female and $32 \%$ male. The mean age of patients was $44.18 \pm 13.51$ years. $66 \%$ patients with cholelithiasis had deranged lipid profile. TC was significantly decreased at one week $(P=0.008)$ and one month $(P=0.00)$ interval after cholecystectomy while TG level was also decreased significantly after one month postoperative compared to values before surgery. There were no significant differences in HDL-C, LDL-c and FBS in the study patients after cholecystectomy. Al was found to be decreased after cholecystectomy.

Conclusion: Cholelithiasis is associated with abnormal lipid profile. Cholecystectomy leads to a significant decrease in some of the parameters of lipid profile and atherogenic index. The presence of gall stones thus should be perceived in the context of metabolic syndrome, which may be investigated and treated. 